\title{
Corrigendum: Ubiquitination of hnRNPA1 by TRAF6 links chronic innate immune signaling with myelodysplasia
}

Jing Fang, Lyndsey C Bolanos, Kwangmin Choi, Xiaona Liu, Susanne Christie, Shailaja Akunuru, Rupali Kumar, Dehua Wang, Xiaoting Chen, Kenneth D Greis, Peter Stoilov, Marie-Dominique Filippi, Jaroslaw P Maciejewski, Guillermo Garcia-Manero, Matthew T Weirauch, Nathan Salamonis, Hartmut Geiger, Yi Zheng \& Daniel T Starczynowski

Nat. Immunol. 18, 236-245; published online 26 December 2016; corrected after print 23 January 2017

In the version of this article initially published online, the 16th author's surname was spelled incorrectly as 'Salamonis. The correct spelling is 'Salomonis. The error has been corrected in the PDF and HTML versions of this article.

\section{Corrigendum: Trans-presentation of IL-6 by dendritic cells is required for the priming of pathogenic $\mathrm{T}_{\mathrm{H}} 17$ cells}

Sylvia Heink, Nir Yogev, Christoph Garbers, Marina Herwerth, Lilian Aly, Christiane Gasperi, Veronika Husterer, Andrew L Croxford, Katja Möller-Hackbarth, Harald S Bartsch, Karl Sotlar, Stefan Krebs, Tommy Regen, Helmut Blum, Bernhard Hemmer, Thomas Misgeld, Thomas F Wunderlich, Juan Hidalgo, Mohamed Oukka, Stefan Rose-John, Marc Schmidt-Supprian, Ari Waisman \& Thomas Korn Nat. Immunol. 18, 74-85 (2017); published online 28 November 2016; corrected after print 23 January 2017

In the version of this article initially published, the label for third bar from the left in Figure 4f ('Anti-IL-6a') was incorrect; the correct label is 'AntiIL-6Ra'. Also, in the legend for Figure 4f, the description of the treatment conditions for middle four bars ('medium alone, or LPS and anti-IL-6 (MR16-1 or polyclonal antibody), anti-IL-6R (mAb\#8)') was incorrect; the correct description is 'LPS alone (Medium), or LPS and anti-IL-6Ra (MR16-1), anti-IL-6 (polyclonal antibody or mAb\#8)'. The error has been corrected for the print, PDF and HTML versions of this article.

\section{Erratum: HIV-1 blocks the signaling adaptor MAVS to evade antiviral host defense after sensing of abortive HIV-1 RNA by the host helicase DDX3}

Sonja I Gringhuis, Nina Hertoghs, Tanja M Kaptein, Esther M Zijlstra-Willems, Ramin Sarrami-Fooroshani, Joris K Sprokholt, Nienke H van Teijlingen, Neeltje A Kootstra, Thijs Booiman, Karel A van Dort, Carla M S Ribeiro, Agata Drewniak \& Teunis B H Geijtenbeek

Nat. Immunol. 18, 225-235 (2017); published online 26 December 2016; corrected after print 23 January 2017

In the version of this article initially published, the fifth author's surname was spelled incorrectly (as 'Sarrami-Fooroshani'). The correct spelling is 'Sarrami-Forooshani'. Also, the cells for the third plot in Figure 1d were identified incorrectly as 'Intesrinal DCs'. The correct label is 'Intestinal DCs'. The errors have been corrected in the PDF and HTML versions of this article.

\section{Erratum: Guidance of regulatory T cell development by Satb1-dependent super-enhancer establishment}

Yohko Kitagawa, Naganari Ohkura, Yujiro Kidani, Alexis Vandenbon, Keiji Hirota, Ryoji Kawakami, Keiko Yasuda, Daisuke Motooka, Shota Nakamura, Motonari Kondo, Ichiro Taniuchi, Terumi Kohwi-Shigematsu \& Shimon Sakaguchi

Nat. Immunol. 18, 173-183 (2017); published online 19 December 2016; corrected after print 23 January 2017

In the version of this article initially published, the labels above the plots in Figure $4 \mathrm{~b}$ were incorrect (with 'Open chromatin' above the first two columns and 'Closed chromatin' above the second two columns). The correct labeling is 'Open chromatin' above the first column, 'Closed chromatin' above the second column, 'Open chromatin' above the third column and 'Closed chromatin' above the fourth column. The error has been corrected in the HTML and PDF versions of the article. 